\title{
Rate-sensitive Strain Localization and Impact Response of Carbon Nanotube Foams with Microscale Heterogeneous Bands
}

\author{
Ramathasan Thevamaran ${ }^{1,2,3}$, Jordan R. Raney ${ }^{1,4}$, and Chiara Daraio ${ }^{1,2, *}$ \\ ${ }^{1}$ Division of Engineering and Applied Science, California Institute of Technology, \\ Pasadena, CA 91125. \\ ${ }^{2}$ Department of Mechanical and Process Engineering, Swiss Federal Institute of \\ Technology (ETH Zurich), 8092 Zurich, Switzerland. \\ ${ }^{3}$ Department of Materials Science and Nanoengineering, Rice University, Houston, TX \\ 77005. \\ ${ }^{4}$ School of Engineering and Applied Sciences, Harvard University, Cambridge, MA 02138. \\ *Corresponding Author: daraio@ethz.ch
}

\begin{abstract}
We describe the deformation mechanisms and the bulk dynamic response of vertically aligned carbon nanotube (VACNT) foams comprised of bands of different densities. The density of the bands is controlled during synthesis by varying the flow-rate of the gas feedstock in discrete steps. We show that the impact response of VACNT foams can be distinctively tailored by introducing heterogeneous bands. For example, we demonstrate that this approach can be used to maintain the stress plateau at low stresses over a large range of strains and to disrupt the expected progressive deformation of the sample. These are desirable characteristics for impact and energy absorption applications. The banded VACNT foams exhibit different deformation mechanisms in dynamics compared to those in quasistatic compression, as observed through in-situ high-speed microscopy.
\end{abstract}




\section{Introduction}

Vertically-aligned carbon nanotube (VACNT) foams present interesting mechanical characteristics due to their functionally-graded properties, which arise from variations in CNT diameter [1-3], density [2-7], alignment [8,9], defect density [6,10] and the presence of contaminants [11]. Several synthesis methods have been developed over the last few years to control these parameters across different length scales, allowing the nano-, micro- and macro-structures of the CNT foams to be tuned, ultimately enabling the bulk mechanical properties to be tailored in desired ways [12-15]. When compressed, the VACNT foams undergo strain localization and typically show a characteristic sequential buckling pattern, in which the buckles nucleate at the low-density region of the sample, and sequentially progress one after the other, governed by their intrinsic functional property gradient [16-18]. Buckles form sequentially, while the remaining section of the sample shows no apparent deformation [17-19]. Such a controlled deformation is generally difficult to achieve in other macroscale materials, but it is a desirable characteristic for the design of protective materials, particularly against impacts and vibrations [20].

VACNT arrays with microscale heterogeneities have been synthesized previously $[8,21,22]$ and the role of these microscale heterogeneities in tailoring the location and extent of strain localization during quasistatic loading, and in changing the energy dissipation and impulse in low energy impact have been reported [22]. In this article, we focus on the bulk dynamic response of the VACNT arrays that are excited by impacts. We use a custom-made dynamic testing setup [23] to study the fundamental dynamic deformation mechanisms and demonstrate the presence of rate effects in VACNT foams with structural heterogeneities.

\section{Experimental methods}

\subsection{Sample synthesis}

We synthesized the VACNT foams with microscale heterogeneities using a floating catalyst thermal chemical vapor deposition (tCVD) process [5,22]. The carbon source 
(Toluene) and the catalyst precursor (Ferrocene) were mixed at a $50 \mathrm{ml}: 1 \mathrm{~g}$ ratio to prepare the feedstock solution, and injected (typically at $0.8 \mathrm{ml} \mathrm{min}^{-1}$ ) into the carrier gas, argon $(800 \mathrm{sccm})$. Silicon substrates were placed inside a $\sim 15 \mathrm{~cm}$ heating zone of the horizontal furnace tube for VACNT growth. The CVD furnace was maintained at $827{ }^{\circ} \mathrm{C}$ and atmospheric pressure. The feedstock solution was injected at controlled rates using a syringe-pump system and the input-rate was varied during synthesis to cause heterogeneity in the microstructure. Use of higher input rates, here $5 \mathrm{ml} \mathrm{min}^{-1}$, of feedstock solution for short duration results in growth of low-density bands in the sample (with the bands oriented parallel with the substrate and perpendicular to the direction of nominal CNT alignment) [22]. Additionally, it has been observed that the higher input rates result in lower diameter CNTs compared to the standard rate $(\sim 30 \mathrm{~nm}$ in high inputrate and $\sim 43 \mathrm{~nm}$ in normal input-rate), and more aligned CNT fibers [22].

We synthesized samples consisting of one lower-density band, obtained using a high input rate precursor injection of $5 \mathrm{ml} \mathrm{min}^{-1}$, sandwiched between two bands synthesized with the standard precursor rate of $0.8 \mathrm{ml} \mathrm{min}^{-1}$. These synthesis steps allow the formation of a confined band within the foam's thickness, presenting lower density and a more compliant mechanical response. Of these layered samples, we synthesized two variants: one for which the high input rate was sustained for 2 minutes (named thin-band VACNTs; thickness: $58 \pm 4 \mu \mathrm{m}$ ), and the other for which this rate was used for 6 minutes (named thick-band VACNTs; thickness: 185 $\pm 21 \mu \mathrm{m}$ ). It should be noted that even though the top and bottom bands are synthesized under the same conditions, the section grown after the soft middle band shows lower density and more aligned CNTs compared to the stiffest top band of the sample (as expected given the known gradients of properties in VACNT foams [1-11]). All the heterogeneous VACNT samples were synthesized to a nominal height of $1 \mathrm{~mm}$, and have an overall bulk density of $0.31 \pm 0.01 \mathrm{~g} \mathrm{~cm}^{-3}$ on average (the bulk density was calculated from the measured mass and the volume of each test sample). The test samples were selected to have similar bulk densities since it has been shown previously that the bulk density of the VACNT foams affects the mechanical properties significantly $[5,18,24]$. If one pauses the growth by turning off the injection of the chemical precursors for more than 10-15 minutes between layers, the layers can be 
readily separated [22]. Using this technique, we measured the changes in bulk density of different layers by separately taking the mass of each layer and dividing it by the overall volume (calculated from the length, width, and height measured in scanning electron microscope (SEM)). The middle band was found to be nearly $20 \%$ lower in bulk density than the overall bulk density of the sample. A set of SEM images showing the microstructure of a VACNT foam with one soft middle band is given in Figure 1. For clarity and consistency throughout the text, we denote the three bands by Roman letters, I, II and III, in the order they were synthesized (Note that the tCVD-VACNTs grow in a bottom-up fashion).

Figure 1. (a-c) A sequence of scanning electron microscopy (SEM) images at different magnifications, showing the microstructure of the VACNT foam with one middle soft band. The three density bands are numbered (I, II and III) in the growth order. High resolution SEM images (d-f) show the VACNT microstructure in three bands: (d) band I, (e) band II, and (f) band III.

We extracted the standalone VACNT samples from the substrate using a razor blade for the mechanical characterization. Once the edges that define the cross sectional area of the samples are cut vertically using a razor blade, the sample peel off easily from the substrate with the application of minimal lateral pressure. Due to the robustness of our floating-catalyst grown VACNT foams, extracting the sample using a razor blade in this way does not cause any observable damage to the samples.

\subsection{Mechanical characterization}

To evaluate the dynamic response of the samples, we performed striker impacts at controlled velocities between 0.5 and $7 \mathrm{~ms}^{-1}$, using a custom made testing setup with insitu high speed microscopy [23]. The samples were attached to a flat-plunge striker (7.2 g mass) using double-sided copper tape and allowed to directly impact a force sensor ( $P C B$ Piezotronic) mounted rigidly on a steel block. All the samples were attached to the striker on the softer band (III) side and the stiffest band (I) side was allowed to impact the force 
sensor, to demonstrate the role of the density distribution on the deformation response. We have shown previously that a VACNT foam of $\sim 1 \mathrm{~mm}$ thickness requires approximately $30 \mu$ s to establish dynamic equilibrium, beyond which the measured stresses represent the equilibrated average stress in the sample [23]. Since the impact events occur in the order of a millisecond in our experiments, the sample is compressed under dynamic equilibrium [23]. However, if strain localizes at the interface of the force sensor and the sample when the soft side of the sample is allowed to impact the force sensor, the role of density may not be obvious as it can be due to either the intrinsic density gradient or any strain gradient that may arise before the dynamic equilibrium is reached. If the strain localizes in the low-density region in the current orientation of the sample where the stiffest band side is allowed to impact the force sensor, such a response can be attributed directly to the influence of density, independent of any strain gradient that may arise at the interface of the sample and force sensor at the instance of impact. We measured the dynamic deformation using a geometric moiré transducer, and used a high-speed microscope (Phantom V1610 high-speed camera with Infinity long distance microscope lens) synchronized to the rest of the experimental setup for in-situ visualization and characterization of the complex microscale dynamic deformations. The measured displacement and force-time histories were then used to calculate the dynamic stress-strain responses. The complete description of the experimental setup and the data reduction methodologies can be found in [23]. We also performed quasistatic compression tests on an Instron ElectroPulse E3000 compression testing system with high-speed microscopic visualization to study the quasistatic deformation mechanisms in comparison with the dynamic response.

\section{Results and discussions}

When a VACNT foam sample with a middle soft band is impacted at a low velocity $(<2$ $\mathrm{ms}^{-1}$ ), initially the stress rises linearly up to a $\sim 10 \%$ strain, and then follows on to a plateau regime compressing the sample at very low stress levels ( $\sim 0.2 \mathrm{MPa})$ and finally reaches densification of the soft bands, beyond which the stress rises rapidly (Figure 2 (a)). Here, the densification strain is approximately equal to the height of the bottom (III) and middle (II) bands normalized by the height of the sample, since the stress necessary 
to compress the highest density band (I) is significantly higher. This suggests that the highest density band (I) remains in its pristine state and only the bands III and II are compressed during low velocity impacts. When a sample is impacted at a higher velocity $\left(>2 \mathrm{~ms}^{-1}\right)$, the initial low-stress level continues up to the compression and densification of the stiff bottom and the soft middle bands, followed by the stress rapidly increasing above $10 \mathrm{MPa}$ without significant deformations (strain $<0.1$ beyond the compression of soft middle band) (Figure 2 (b)). This same pattern is followed for very high impact velocities of up to $7 \mathrm{~ms}^{-1}$ (see the SI Figure 1 in supplementary information for representative dynamic stress-strain responses at different velocities). We compare these dynamic stress-strain responses to the quasistatic stress-strain response of a similar VACNT sample with heterogeneous bands (Figure 2 (b)). The sample exhibits much stiffer response and a much larger hysteresis in quasistatic compression compared to the dynamic compression, suggesting the significant role of response timescales. In dynamics, once the buckle instability occurs, the sample continues to deform at low stress-levels without following the typically expected sequential progressive buckling path with local softening and stiffening. This is evident from the long stress-plateau at very low stress levels in dynamics compared to the quasistatic compression (Figure 2 (b)).

Figure 2. Stress-strain responses of VACNT foams with heterogeneous bands (with one soft middle band): (a) the dynamic stress-strain response of a sample impacted at 0.85 $\mathrm{ms}^{-1}$, (b) the dynamic stress-strain response of a sample impacted at $2.15 \mathrm{~ms}^{-1}$ compared to the low-velocity impact response in (a), and the quasistatic stress-strain response of a sample.

To quantitatively analyze the dynamic responses, we plotted the peak stress $\left(\sigma_{p}\right)$ attained during impact, and the energy dissipated ( $\left.W_{\text {dissipated }}\right)$ through the loading-unloading hysteresis with increasing impact velocities (Figure 3 (a), (b)). For both continuous and banded samples, the peak stress increases with increasing impact velocity, due to the intrinsic density gradient along the height of the samples, and the densification of the buckled folds during compression [18]. The hysteretic energy dissipation also increases 
with increasing impact velocity (as measured by the increasing area of the stress-strain hysteresis). Both types of samples show similar stress and energy dissipation as a function of impact velocity, with the benefit provided by the banded samples being the higher strain reached prior to densification.

Figure 3. Comparison of dynamic response between VACNT foams with heterogeneous bands (Banded VACNT) and VACNT foams without heterogeneous bands (Continuous VACNT Foam [18]): (a) the variation of peak stress with impact velocity, (b) the variation of hysteretic energy dissipated with impact velocity, the variation of dynamic cushion factor with (c) maximum strain and (d) impact velocity, and the stress-strain responses showing the effects of the heterogeneous bands at (e) a low velocity impact $(<1$ $\left.\mathrm{ms}^{-1}\right)$, and (f) a high velocity impact $\left(\sim 4.5 \mathrm{~ms}^{-1}\right)$. Both the continuous VACNT foams and the VACNT foams with heterogeneous bands compared here have similar bulk densities.

To evaluate the impact performance, we calculated the dynamic cushion factor $\left(C_{d y n}\right)-$ the peak stress $\left(\sigma_{p}\right)$ normalized by the energy absorbed up to the peak stress $\left(W_{p}\right)$-and plotted it against the varying maximum strain reached $\left(\varepsilon_{\max }\right)$ during impact (Figure $3(\mathrm{c})$ ), combining the three critical parameters in impact response. The trend in cushion factor for our samples is unique compared to conventional foam materials [25] and the continuous VACNT foams (with no heterogeneous bands) [18]. We have also provided the dynamic cushion factor as a function of impact velocity for direct comparison (Figure 3 (d)). The cushion factor is very low for low velocity impacts and then exhibits a sharp rise above a certain impact velocity $\left(\sim 2 \mathrm{~ms}^{-1}\right)$, followed by a decreasing trend similar to that of other foam materials [25]. This response arises due to the controlled deformations achieved in the VACNT foams with heterogeneous bands, where only the softer bands deform during the low velocity impacts exhibiting low-cushion factors, and a sudden increase of cushion factor occurs at $\sim 2 \mathrm{~ms}^{-1}$ impacts when the soft bands are compressed fully and the deformation progresses further into the stiffest band. When the samples are impacted at much higher velocities, the response of the stiffest band dominates the bulk 
behavior and the cushion factor decreases following a similar decreasing trend with impact velocity like that of a continuous VACNT foam (Figure 3 (d)). It should be noted that in general the cushion factors are calculated from quasistatic stress-strain curves and plotted against the peak stress to evaluate the cushioning ability of a material at a given peak stress level [25]. A similar cushion factor curve plotted against the peak stress for the dynamic compression is given in SI Figure 2.

As shown in Figure 3 (a), all VACNT foams exhibit similar peak stresses for a given impact velocity. The continuous VACNT foams dissipate slightly higher energy through hysteresis in an intermediate velocity range between 2 and $6 \mathrm{~ms}^{-1}$ and the energy dissipation is comparable for both foams bellow and above this range of velocities (Figure 3 (b)). This response leads to a lower dynamic cushion factor of the continuous VACNT foams in the velocity range of 2-6 $\mathrm{ms}^{-1}$ (Figure 3 (c), (d)), which is typically associated with desirable metrics of impact performance, such as higher energy absorption and lower transmitted stress amplitude. The VACNT foams with heterogeneous bands perform comparable to continuous VACNT foams in low velocities due to the controlled deformations up to the soft middle band, and also at higher velocities $\left(>6 \mathrm{~ms}^{-1}\right)$ through the deformation of undeformed-sections of the stiffest band. As shown in the dynamic stress-strain responses in Figure 3 (e) and (f), though adding softer bands to the VACNT foams may decrease the overall hysteretic dissipation, it has the benefit of providing a lower and more uniform plateau stress profile, which enables high strain deformation without the foam exceeding the same level of transmitted stress observed for continuous VACNTs. Such a long stress-plateau at lower stress levels of banded-VACNT foams compared to continuous VACNT foams has also been shown to exist in quasistatic compression (see the supplementary information of [22]), however without the drastic differences seen in the present study (dynamics).

Additionally, we examined the effect of the soft bands on the material response when the same sample is subjected to multiple impacts (see SI Figure 3 in supplementary information). It is known that VACNT foams subjected to repeated loading exhibit a preconditioning effect, involving a large decrease in hysteresis area over the first few loading cycles but also an eventual steady-state response that can continue for all future 
cycles indefinitely after this [16]. This preconditioning effect has been shown to be associated with localized buckling, such that when the strain is allowed to increase beyond the maximum strain of earlier loading cycles the material behaves as if it had not been compressed previously, exhibiting a behavior analogous to the Mullins effect [26]. Due to these preconditioning effects and the resultant reduction in hysteresis loop, when the VACNT foams are repeatedly impacted at the same striker velocity, the strain increases for consecutive cycles [SI Figure 3(a)]. When the striker velocity is increased, the strain-dependent nature of the preconditioning becomes more apparent [SI Figure 3(b)]. It is interesting to note that once preconditioning has occurred and a steady-state response has been reached, the soft band no longer has an effect and the response becomes similar to that of a continuous VACNT foam.

To understand the effect of the thickness of the soft middle band on the fundamental dynamic response of the VACNT foams with heterogeneous bands, we characterized the dynamic response of samples with the middle soft band synthesized for 2 min and 6 min, at comparable impact velocities. An increased duration of the higher precursor input rate results in a soft middle band with higher thickness and more gradual variation in the microstructure [22]. The variation of the thickness of the soft middle band as a function of the duration of synthesis step is shown in SI Figure 4 (the band thickness was measured in SEM). The average thicknesses of the bands are $58 \pm 4 \mu \mathrm{m}$ and $185 \pm 21 \mu \mathrm{m}$ for the two and six minute synthesis steps, respectively. We also used the response of continuous VACNT foams with no heterogeneous bands as a benchmark. The VACNT foams with a thick soft middle band exhibits a response that is more similar to the response of the continuous VACNT foams with no heterogeneous bands. They also exhibit significantly lower peak stresses compared to the VACNT foams with thin soft middle bands. This response may be a result of gradual variation in the microstructure from the soft band to the band synthesized after the soft band, rather than the abrupt changes that could have been induced during the short feedstock input (2 min) [22].

Figure 4. Comparison of the dynamic stress-strain responses of VACNT foams: (i) with 
a soft band synthesized for 2 min (thin-band VACNTs), (ii) a soft band synthesized for 6 min (thick-band VACNTs) and (iii) with no heterogeneous bands (continuous VACNTs).

We used high-speed microscopy to visualize the deformation mechanisms in situ, during dynamic compression of the samples. Snapshots from the high-speed microscopy and the corresponding stress-strain response for VACNT foam with a soft middle band, impacted at $0.85 \mathrm{~ms}^{-1}$ are shown in Figure 5 (also in Supplementary Video 1). The soft middle band (II) appears darker than the other two sections as indicated on image (1) of Figure 5. It should be noted that the sample appears inverted in the images-i.e. the section grown last (III), which is found near the substrate in the as-grown sample, is attached to the striker. It has been shown previously for the range of impact velocities discussed here that the formation and progression of buckles in VACNT foams (with no heterogeneous bands) are always governed by the intrinsic density gradient, regardless of which side is impacted [18]. Therefore, to demonstrate the predominant role of the density, we attached the sample to the striker such that the side of the stiffest band (I) impacts the force sensor directly.

Figure 5. Snapshots from the high-speed microscopy showing the deformation mechanisms found in the dynamic compression of the VACNT foams with a middle soft band. The stress-states corresponding to the deformations shown in the images are indicated as (1-8) on the dynamic stress-strain diagram. The sample was impacted at 0.85 $\mathrm{ms}^{-1}$.

When the sample is impacted, the deformation localizes first at the section adjacent to the striker (in band III), which is the weakest section of the sample as the final CNT growth occurs at the substrate interface during the termination of synthesis (Image 2 of Figure 5). The deformation progresses compressing that section of the sample as the stress rises linearly. Following the linear regime, the stress progresses into a plateau regime as global buckling of the section occurs as indicated on image 3 of Figure 5. Until this instance, we do not notice any strain localization in the soft middle band (II), and it deforms only after the bottom band (III) is compressed. Stress rises moderately until both the bottom whole 
section (III) and the soft middle band (II) are compressed all the way up to the stiffest band (I), and then rapidly increases as those two sections (III and II) are densified. After reaching the peak stress, the striker unloads as the sample recovers. The stress rapidly decreases during the initial unloading and then slowly reaches zero as the sample recovers more than $95 \%$ of its deformation at the end of unloading. The recovery of all the samples tested in velocities between 0.5 and $7 \mathrm{~ms}^{-1}$ is $93.7 \pm 4.2 \%$ on average. When samples are impacted at very high velocities, the stiffest band (I) starts deforming with characteristic progressive buckling, once the other two softer bands are completely deformed and densified (Supplementary Video $2\left(6.81 \mathrm{~ms}^{-1}\right.$ impact)).

Figure 6. The stress-strain response and deformation mechanisms during quasistatic compression of a VACNT foam with heterogeneous bands (1-soft middle band). The stress-states corresponding to the microscope images are indicated as (1-6).

We found that these deformation mechanisms in dynamic compression are very different from those of the quasistatic compression, found in the present study (Figure 6) and in a previous study [22]. In the previous study, it was shown that the soft bands collapse predominantly when a VACNT foam with multiple heterogeneous bands is subjected to static compression in a custom-made vise (Figure 2 of [22]). When we performed in-situ microscopy during a quasistatic compression test, we observed (Image (2) of Figure 6) that the strain localizes first at the bottom region (band III) that was grown before the termination of the synthesis (the region of the sample that was adjacent to the substrate before extraction for mechanical characterization), similar to the dynamic case (Image (2) of Figure 5). Then the localization occurred in the soft middle band (Image (3) of Figure 6 ), instead of progressing from the initially buckled section. This controlled localization in the soft middle band is similar to what has been shown in Ref. [22], in static compression, but distinctively different from what we observed in dynamic compression - where a continuous progression of the deformation occurs starting from the location where the initial deformation nucleates. During this deformation, stress rises 
almost linearly with strain. Subsequently, the bottom band undergoes buckling as a whole during which the stress deviates from the linear trend and shows a nonlinear rise in strain (Figure 6). The sample is compressed up to $50 \%$ strain in this experiment during which the stiffest band (I) didn't show any observable deformation. During unloading, the sample recovers $73 \%$ of its strain, which is, although a significant recovery, much less compared to the recovery during dynamic compression ( $94 \%)$. Lower recovery in lower strain rates has been reported previously also [18,27], and often attributed to the effects of the surface roughness and lack of stored elastic energy to overcome the van der Waal's interactions that occurs during the formation of buckles.

\section{Conclusions}

In summary, we performed impact experiments on VACNT foams with heterogeneous bands to understand the fundamental role of the microscale heterogeneities on the bulk dynamic response and the deformation mechanisms. We found that the VACNT foams with heterogeneous bands exhibit a stress-strain response with well-defined linear, plateau and densification regimes when impacted at low-velocities $\left(<2 \mathrm{~ms}^{-1}\right)$. When impacted at higher velocities, the samples deform at very low stress levels up to the complete deformation and densification of the bottom and middle soft bands and then the stress rapidly increases with small strains as the stiffest-band starts deforming. We also showed that the stress-strain responses and the deformation mechanisms in dynamics are significantly different from that found in the quasistatic compression. During dynamic compression, the deformation always localizes at the bottom low-density region of the sample and progressed towards the soft middle band. The soft middle band acts like a deformation-arrest barrier that prohibits further progression of the deformation, unless

impacted at very high impact velocities. In contrast, during quasistatic compression, the strain localizes in the softest sections and slowly progressed to the rest of the samples. We also showed that the stress-strain response could be significantly tailored when the thickness of the soft middle band is increased. Our studies show that the microstructure of the VACNT foams can be engineered to achieve desirable deformation mechanisms and to tailor the stress-strain response to meet the needs of different protective 
applications that require materials exhibiting low weight, high energy absorption, and high strain recovery.

\section{Acknowledgments}

This work was supported by the Institute for Collaborative Biotechnologies (ICB) under the contract W911NF-09-D-0001 with the Army Research Office (ARO). The authors thank Mr. Jake Christoph for synthesizing supplemental samples related to this study in the laboratory of Prof. David Jack.

\section{References}

[1] Li X, Zhang X, Ci L, Shah R, Wolfe C, Kar S, et al. Air-assisted growth of ultralong carbon nanotube bundles. Nanotechnology 2008;19:455609.

[2] Yaglioglu O, Cao A, Hart AJ, Martens R, Slocum AH. Wide range control of microstructure and mechanical properties of carbon nanotube forests: A comparison between fixed and floating catalyst CVD techniques. Adv Funct Mater 2012;22:5028-37.

[3] Nessim GD, Hart AJ, Kim JS, Acquaviva D, Oh J, Morgan CD, et al. Tuning of vertically-aligned carbon nanotube diameter and areal density through catalyst pretreatment. Nano Lett 2008;8:3587-93.

[4] Pushparaj VL, Ci L, Sreekala S, Kumar A, Kesapragada S, Gall D, et al. Effects of compressive strains on electrical conductivities of a macroscale carbon nanotube block. Appl Phys Lett 2007;91.

[5] Raney JR, Misra A, Daraio C. Tailoring the microstructure and mechanical properties of arrays of aligned multiwall carbon nanotubes by utilizing different hydrogen concentrations during synthesis. Carbon 2011;49:3631-8.

[6] Li Y, Kang J, Choi J-B, Nam J-D, Suhr J. Determination of material constants of vertically aligned carbon nanotube structures in compressions. Nanotechnology 2015;26:245701.

[7] Pathak S, Mohan N, Decolvenaere E. Local relative density modulates failure and strength in vertically aligned carbon nanotubes. ACS Nano 2013:8593-604.

[8] Pinault M, Pichot V, Khodja H, Launois P, Reynaud C, Mayne-L'Hermite M. Evidence of sequential lift in growth of aligned multiwalled carbon nanotube multilayers. Nano Lett 2005;5:2394-8. 
[9] Pathak S, Raney J, Daraio C. Effect of morphology on the strain recovery of vertically aligned carbon nanotube arrays: an in situ study. Carbon 2013;1:1-22.

[10] Chakrabarti S, Gong K, Dai L. Structural evaluation along the nanotube length for super-long vertically aligned double-walled carbon nanotube arrays. J Phys Chem C 2008;112:8136-9.

[11] Malek Abbaslou RM, Soltan J, Dalai AK. The effects of carbon concentration in the precursor gas on the quality and quantity of carbon nanotubes synthesized by CVD method. Appl Catal A Gen 2010;372:147-52.

[12] Raney JR. Hierarchical structures of aligned carbon nanotubes as low-density energy-dissipative materials. California Institute of Technology Thesis, 2012.

[13] Coleman JN, Khan U, Blau WJ, Gun'ko YK. Small but strong: A review of the mechanical properties of carbon nanotube-polymer composites. Carbon 2006; $44: 1624-52$.

[14] Liu L, Ma W, Zhang Z. Macroscopic carbon nanotube assemblies: Preparation, properties, and potential applications. Small 2011;7:1504-20.

[15] Di J, Wang X, Xing Y, Zhang Y, Zhang X, Lu W, et al. Dry-processable carbon nanotubes for functional devices and composites. Small 2014:1-20.

[16] Cao A, Dickrell PL, Sawyer WG, Ghasemi-Nejhad MN, Ajayan PM. Supercompressible foamlike carbon nanotube films. Science 2005;310:1307-10.

[17] Yaglioglu O. Carbon nanotube based electromechanical probes. MIT Thesis 2007.

[18] Thevamaran R, Meshot ER, Daraio C. Shock formation and rate effects in impacted carbon nanotube foams. Carbon 2015;84:390-8.

[19] Hutchens SB, Hall LJ, Greer JR. In situ mechanical testing reveals periodic buckle nucleation and propagation in carbon nanotube bundles. Adv Funct Mater 2010;20:2338-46.

[20] Sun G, Li G, Hou S, Zhou S, Li W, Li Q. Crashworthiness design for functionally graded foam-filled thin-walled structures. Mater Sci Eng A 2010;527:1911-9.

[21] Jackson JJ, Puretzky AA, More KL, Rouleau CM, Eres G, Geohegan DB. Pulsed growth of vertically aligned nanotube arrays with variable density. ACS Nano 2010;4:7573-81.

[22] Raney J, Wang R, Daraio C. Control of microstructural heterogeneities in carbon nanotube foams. Carbon 2012;52:193-200. 
[23] Thevamaran R, Daraio C. An experimental technique for the dynamic characterization of soft complex materials. Exp Mech 2014;54:1319-28.

[24] Misra A, Raney JR, Craig AE, Daraio C. Effect of density variation and noncovalent functionalization on the compressive behavior of carbon nanotube arrays. Nanotechnology 2011;22:425705.

[25] Gibson L, Ashby M. Cellular solids. second. Cambridge University Press; 1999.

[26] Raney J, Fraternali F, Daraio C. Rate-independent dissipation and loading direction effects in compressed carbon nanotube arrays. Nanotechnology 2013;24.

[27] Pathak S, Lim EJ, Abadi PPSS, Graham S, Cola BA, Greer JR. Higher recovery and better energy dissipation at faster strain rates in carbon nanotube bundles: An in-situ study. ACS Nano 2012;6:2189-97. 

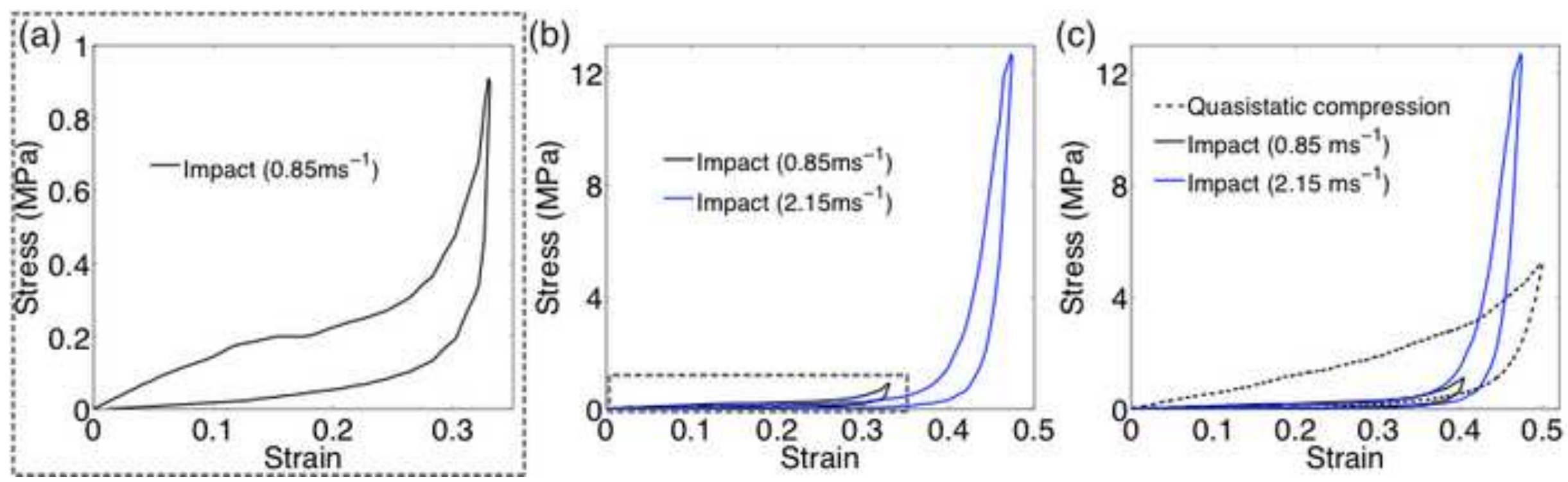
(a)

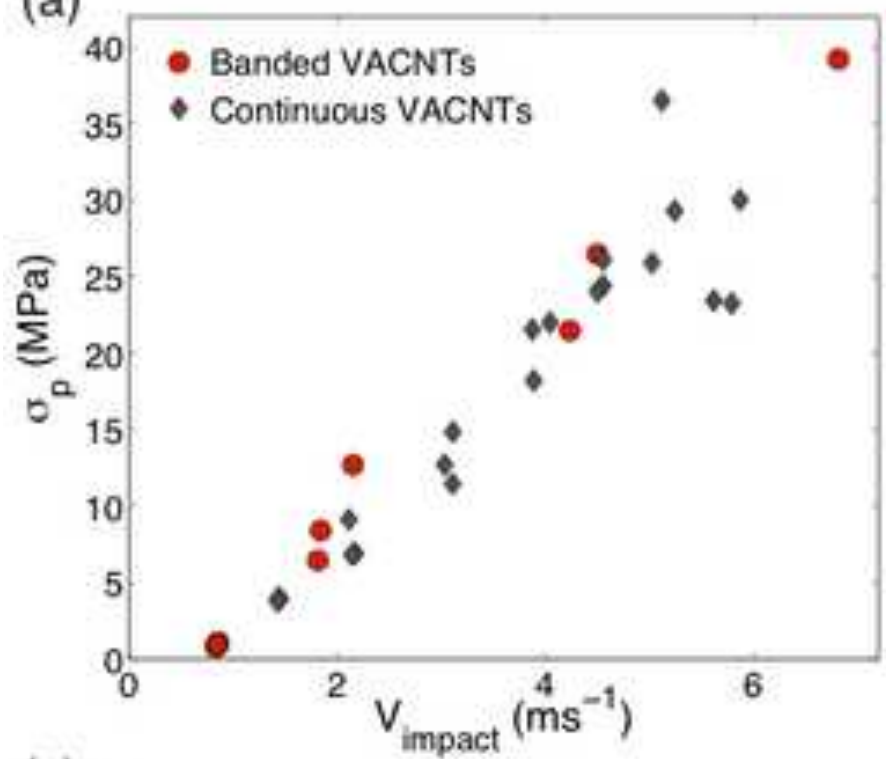

(c)
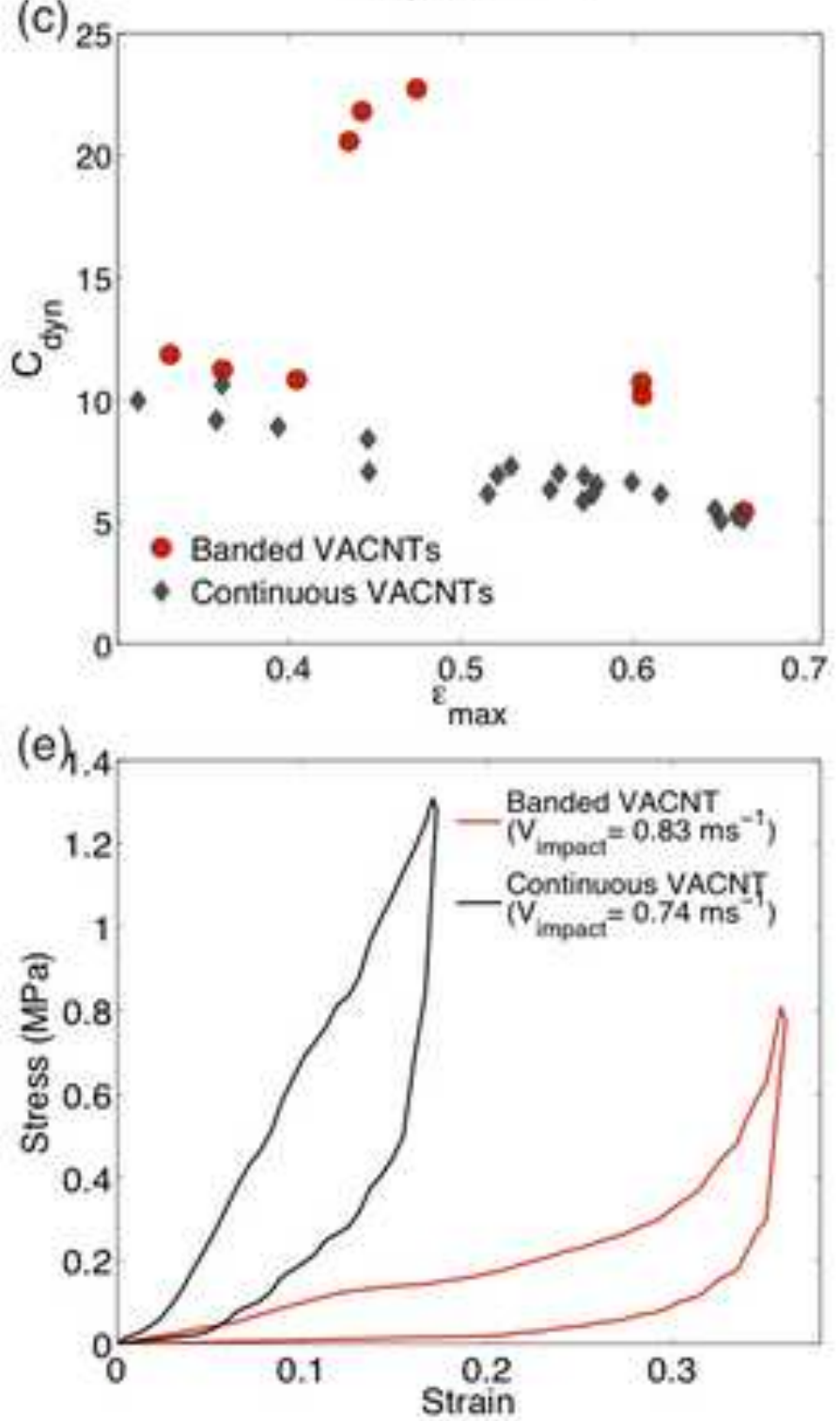

(b)
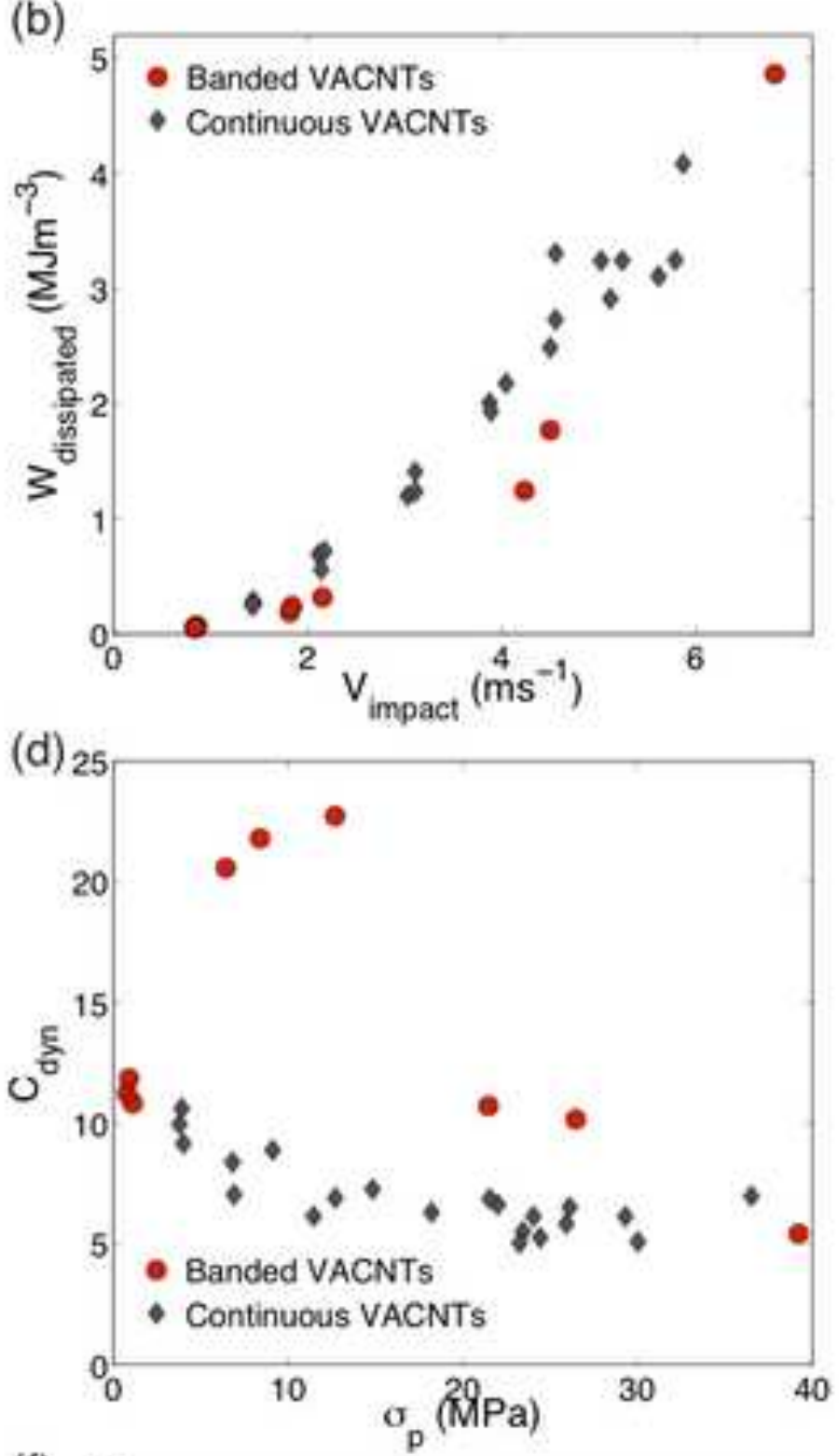

(f)

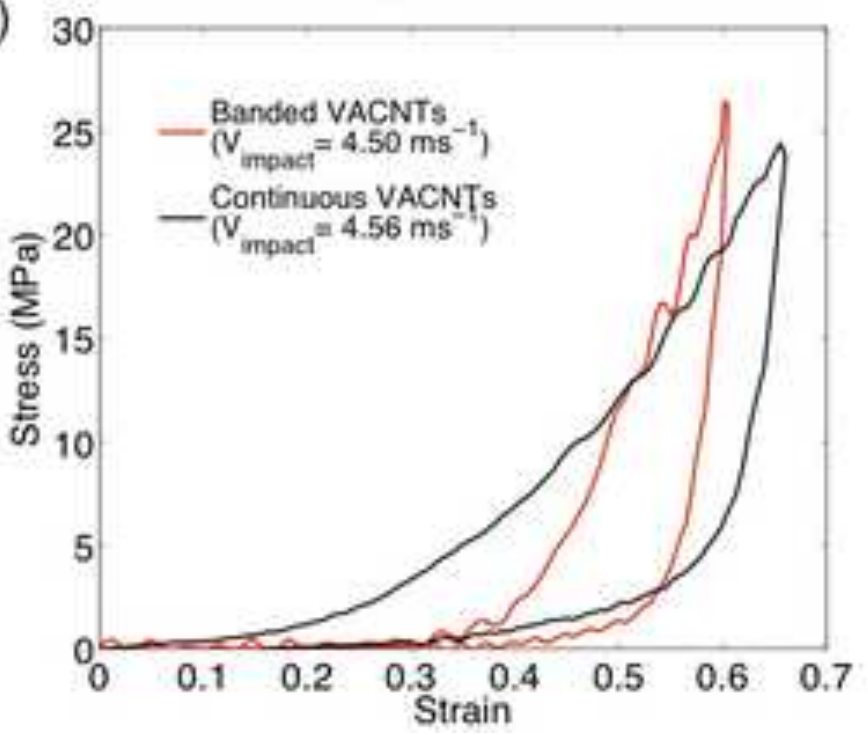




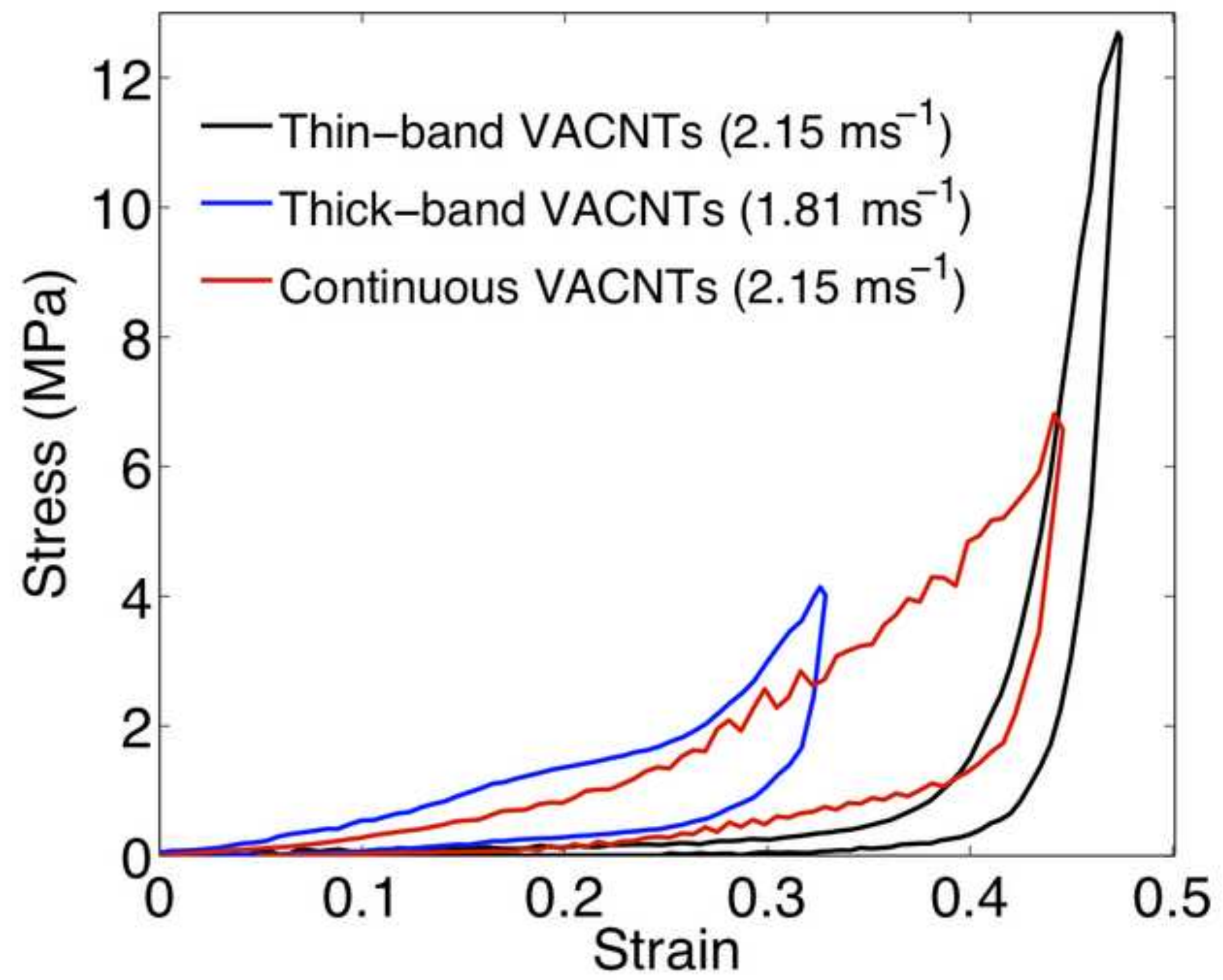




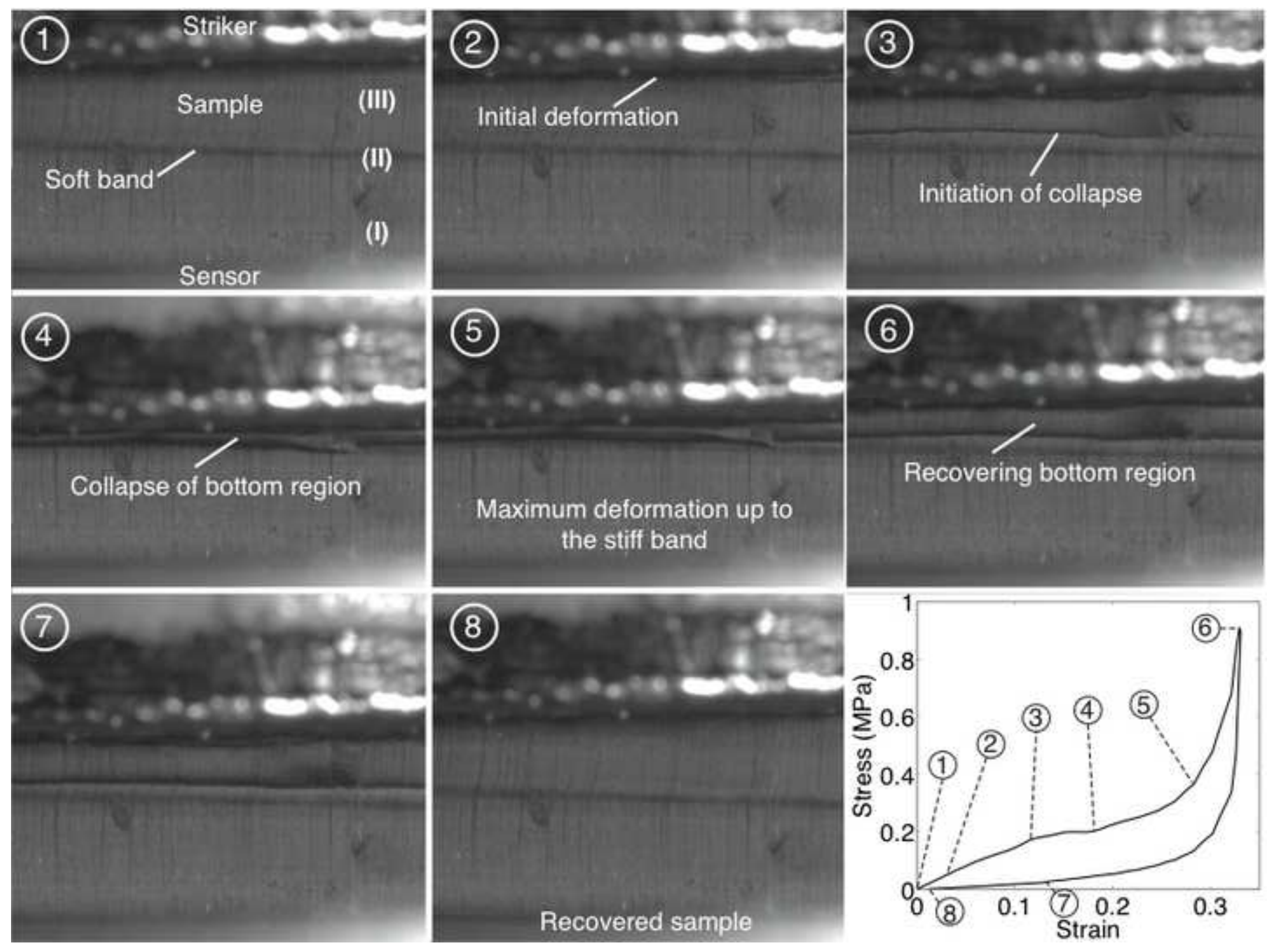



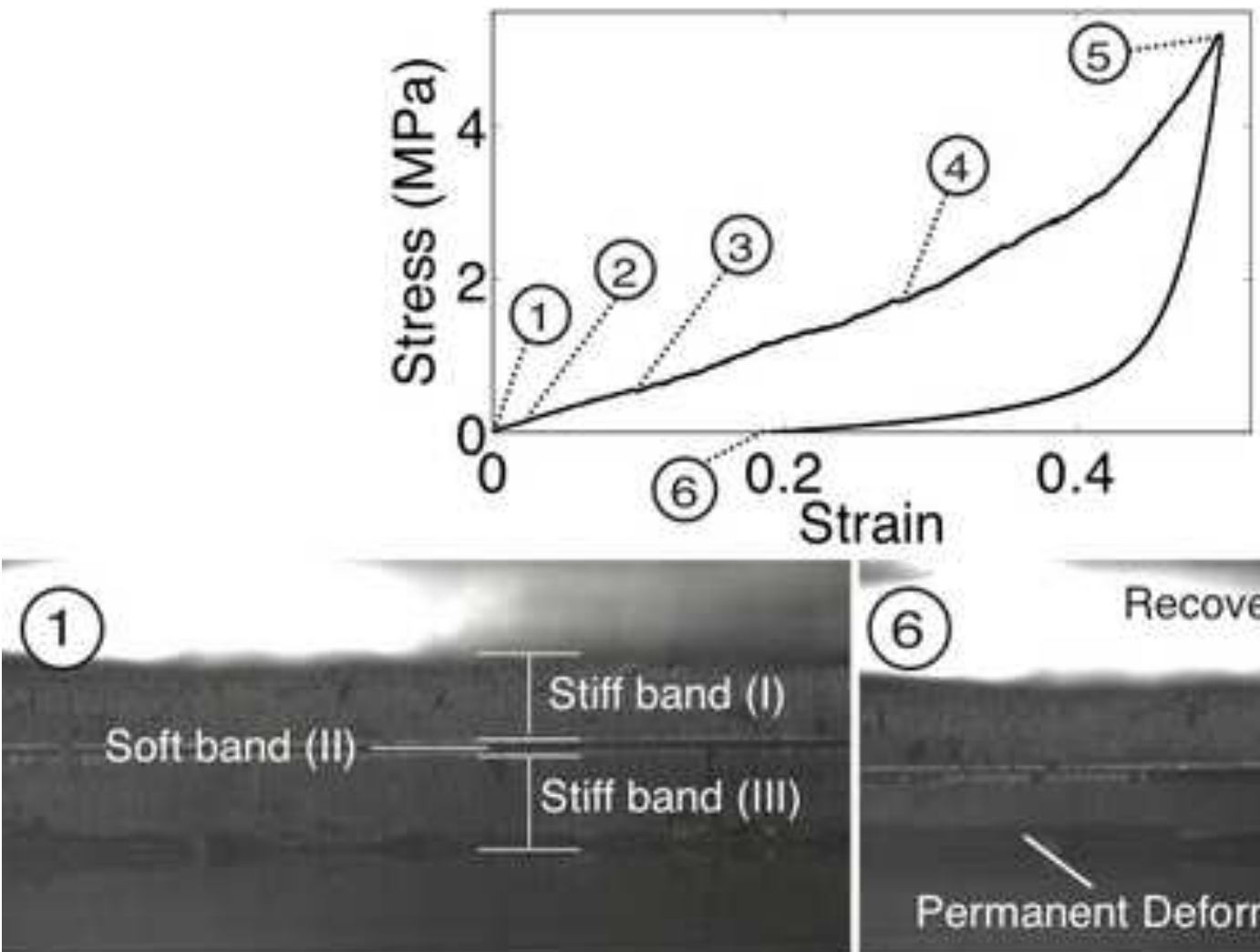

Recovered specimen

(2)

(5)

Initial buckle

Permanent Deformation

\section{At $50 \%$ compression}
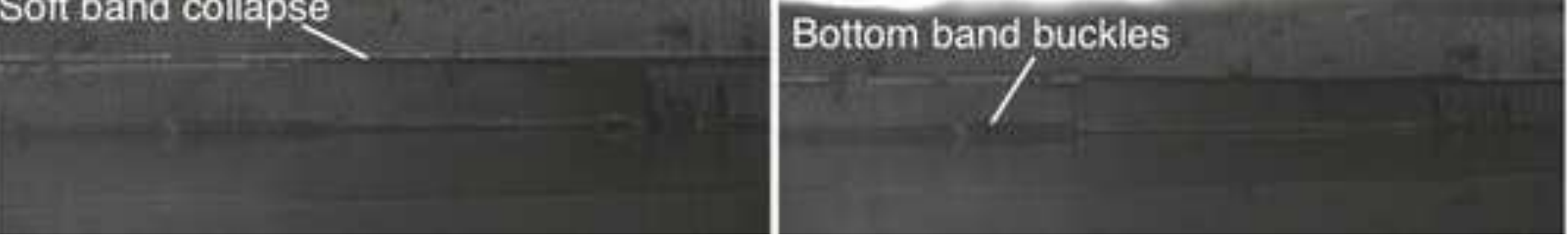